\title{
Justification of Spatially-Planned Solutions and Determination of the Dimension Block in the Working of the Medium Thick Inclined Coal Seams with the Room and Pillar System
}

\author{
Thang Pham Duc ${ }^{1, *}$, Anh Phan Tuan ${ }^{1}$, Viktor Vitcalov ${ }^{1}$, and Phuc Le Quang ${ }^{2}$ \\ ${ }^{1}$ Mining Institute, National University of Science and Technology MISiS, 119049, Leninsky Av., 4, \\ Moscow, Russia \\ ${ }^{2}$ Saint-Petersburg mining University, 99106, Saint-Petersburg, 2 21st line of Vasilyevsky island, \\ Russian Federation
}

\begin{abstract}
Technological parameters for working out of the medium thick inclined coal seams with a diagonal arrangement of a line of a working face. An analysis of the constraints that apply the technology and the complexities of the mining and geological conditions of the Quang Ninh coal basin. The possibility and expediency of using the foreign experience of working out of the medium thick inclined coal seams in the mines of Vietnam and justification of spatially-planned solutions for working. The scheme of preparation and working out of the excavation area is given in view of the technological features in difficult mining and geological conditions, using the room and pillar system and determination of the rational length of the block along the strike.
\end{abstract}

\section{Introduction}

Based on the analysis of advanced technologies for the working of gently and inclined coal seams of medium thickness used in coal mines in the countries with a developed mining industry, it can be stated that up to the present time no modern technological schemes, as well as means for extracting coal and timbering the face for working inclined coal seams of medium thickness, and the use of the developed technologies does not give a tangible effect, due to the lack of scientifically sound design and engineering solutions associated with complex specific mining and geological and mining conditions of coal bedding $[1,2$, 3].

In the advanced mining countries of the world, such as Australia, South Africa, the United States, despite of the widespread use of longwall mining technologies, room and pillar systems in the overall structure of underground mining occupy more than $40 \%$ [1]. About $65 \%$ of USA production falls under the room and pillar system, in South Africa $92.5 \%$. The wide application of the room and pillar mining system in the USA and South

* Corresponding author: phamducthangmet@gmail.com 
Africa is explained by the lower initial costs for the purchase of mining equipment in comparison with the equipment for longwall and its high mobility [3, 4].

Currently, in Russia, as well as in other coal mining countries, for the development of coal deposits with high technical and economic indicators, the main direction is the use of longwall systems characterized by mining operations in longwall with the use of mechanized complexes equipped with the mobile hydraulic support. However, the field of application of mechanized complexes with the length of the faces is considerably limited by mining and geological conditions and is effective only when operating under favourable conditions. In other cases, depending on complicated mining and geological conditions, complicated configuration of sites, complicated structures, limited in strike and dip, must be practised with highly effective non-traditional and room and pillar technology $[5,6]$.

In connection with earlier studies, considerable coal reserves (within 67\%) in the Quang Ninh coal basin are located in the geological disturbance zones and in areas of minefields with limited strike sizes, which it is advisable to work with the room and pillar mining system [7]. The main advantage of this technology is its ability to adapt to complicated mining and geological conditions of bedding of coal seams and limited technological parameters of the excavation field that are not acceptable for excavating longwall systems. So justification of spatially-planned solutions and determination of the rational length of the block along the strike for expansion to provide safety and efficiency in the working are necessary.

\section{Justification of spatially-planned solutions}

In recent years, scientific research has been carried out at the mines of Vietnam on the introduction of room and pillar mining system with coal cutting by drilling and blasting, which is increasingly used in the mines of the Quang Ninh coal basin. The advantage of this technology is - low initial costs and low cost of coal in comparison with the longwall technologies. The main disadvantage of the room and pillar system with the extraction of the rib pillar by drilling and blasting is large losses of coal in the pillars, which sometimes can reach $30-40 \%[7,8,9,10]$.

At the Uong Bi Mine, an experimental industrial study of the technology for working out the seam 8 was carried out using a room and pillar mining system. Within the minefield, the coal seam 8 is disturbed by numerous faults, the number and amplitude of which increases with depth. The thickness of the seam ranges from 1.2 to $6.22 \mathrm{~m}$, the average thickness is $3.5 \mathrm{~m}$, the angle of incidence is 30 to 45 degrees, and in some areas, it reaches up to 50 degrees. The coal layer is of complicated structure, there are interlayers of clay shale, and splitting into separate packs [11].

The development of the block (or strip) for the room and pillar system begins with the carrying out of drifts and chutes. The distance between the drifts is determined by the height of the horizon (or floor). From the conveyor drift at the border of the strip, rise up parallel excavations at an angle of 30 degrees (diagonal chute). For airing the chutes and room, as well as the delivery of materials and equipment, the chutes are connected by the heading cross. The technological scheme of preparation of conducting of mining works with the application of the room and pillar system has resulted in 'Figure 1'.

For the room and pillar mining system, which is being tested in the mines of the Quang Ninh coal basin, drifts and chutes are attached to the trapeziform wooden support.

Within the block, the mining of the coal seam is carried out, from the block boundary to the coal chute in descending order. The width of the room is 4 to $5 \mathrm{~m}$ and the rooms are made at an angle of 20 degrees to the horizontal for the purpose of facilitating the transport of coal through the enamelled pans, the rib pillar with the width 8 to $10 \mathrm{~m}$ is withdrawn in reverse, leaving the protective coal pack (protective coal pillar) to prevent penetration of 
the collapsed rocks into the face. In the case of simultaneous holding of rooms between two rib pillars, it is necessary to keep an advancing of at least $15 \mathrm{~m}$ between yourself.

For the transportation of coal in a diagonal room and chutes, enamelled pans are used. Coal, repulsed by drilling and blasting, is directed along enamelled pans to the chute and along with it to the conveyor drift and then to the haulage crosscut to the surface.

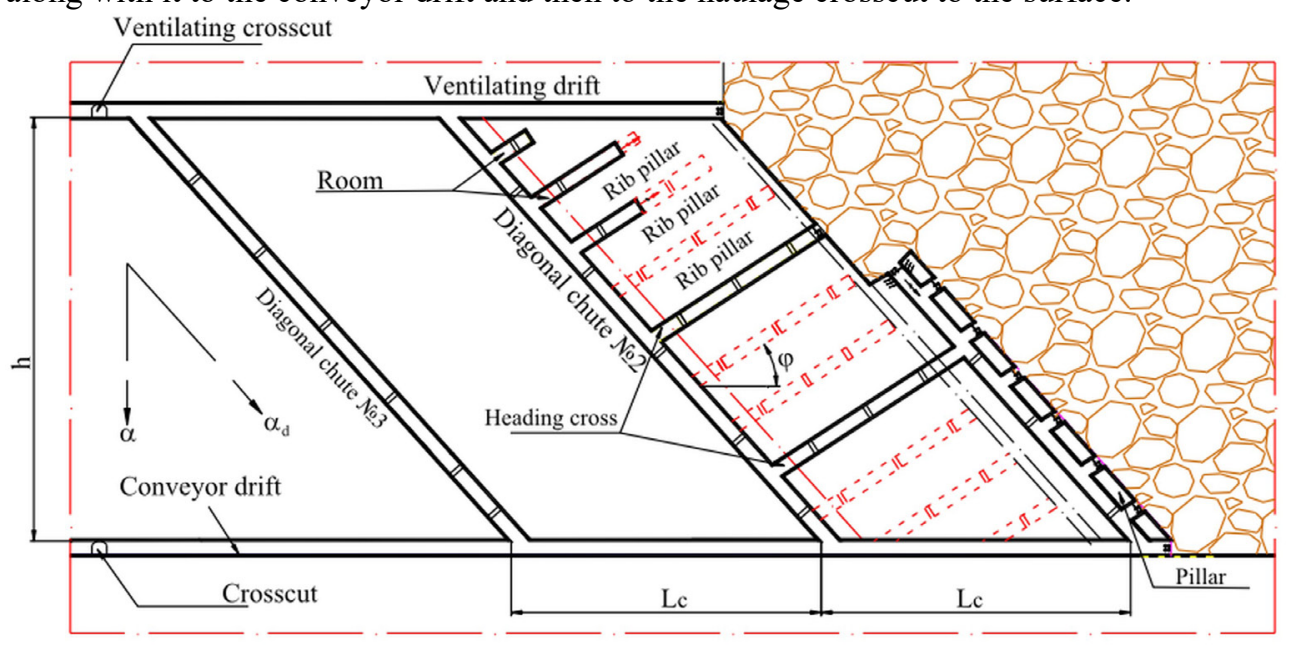

Fig. 1. Technological scheme for the preparation of the room and pillar system with diagonal chute.

When chutes and diagonal room are used, ventilation is effected by the fans of local airing, and when the rib pillars are extinguished, ventilation is carried out to offset the general depression.

To substantiate the optimal turning angle of mine workings, depending on the angle of dip, and to derive analytic expressions reflecting the relationship between the change in the angle of inclination of the coal seam along the mine workings $\left(\alpha_{d}\right)$ and from the angle of the diagonal position of the mine workings $(\beta)$ at various angle of dip of coal seam, a schematic diagram is drawn up and considered (Figure 2) and an expression is constructed to ensure the connection between the " $\alpha$ and $\alpha_{d}$ ", which will have the following form:

$$
\sin \alpha_{d}=\sin \alpha \cdot \sin \beta
$$

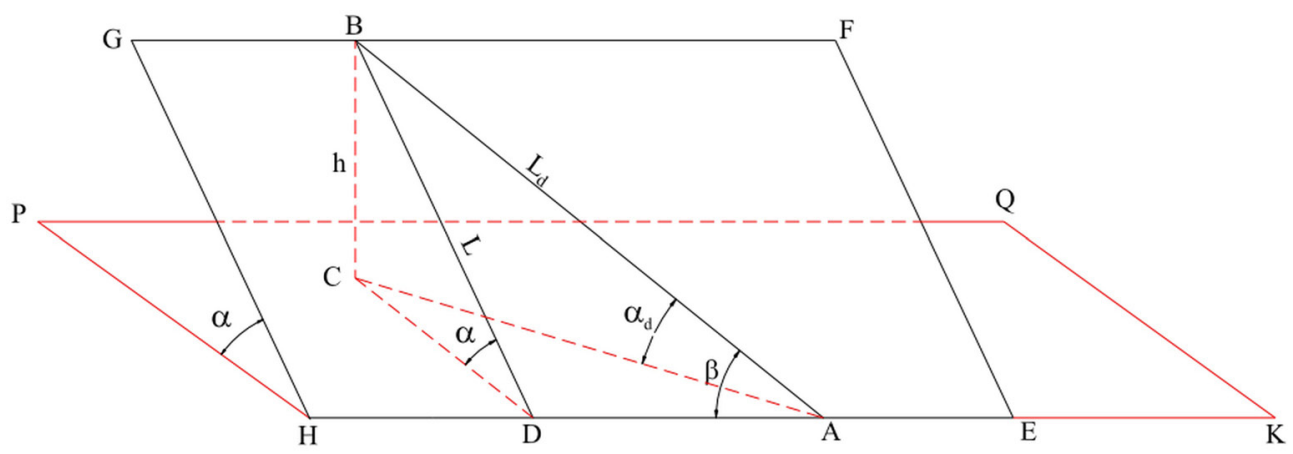

$\alpha$ - angle of dip; $\alpha d$-angle of slope of mine workings; $\beta$ - angle of diagonal position of mine workings

Fig. 2. Scheme for calculating the decrease in the angle of inclination of the mine workings.

On the basis of the obtained analytical expression (1) and the assumed angles of dip $\alpha$ $(20,25,30,35$ degrees), the characteristic curves reflecting changes in the angle of 
inclination of the coal seam along the mine workings $\alpha_{d}$, from the angle of diagonal position of mine workings $\beta$, which are shown in 'Figure 3'.

At an angle of dip of the coal seam from 30 to $35^{\circ}$, the diagonal chute should be drawn at an angle of $25^{\circ}$ with respect to the horizon, and the angle of the diagonal position of the mine workings will be $58^{\circ}$ (at the angle of dip of the formation $\alpha=30^{\circ}$ ) or $47^{0}$ (at the dip angle of the formation $\alpha=35^{\circ}$ ).

On the basis of established regularities, changes in the diagonal position of the mine workings (chutes, headings), depending on the angle of dip of the coal seam, allow spatial planning decisions to be made to cut the floor, in order to improve the efficiency of the excavation blocks, depending on the adopted technological scheme depending on mining and geological conditions and the means of integrated mechanization.

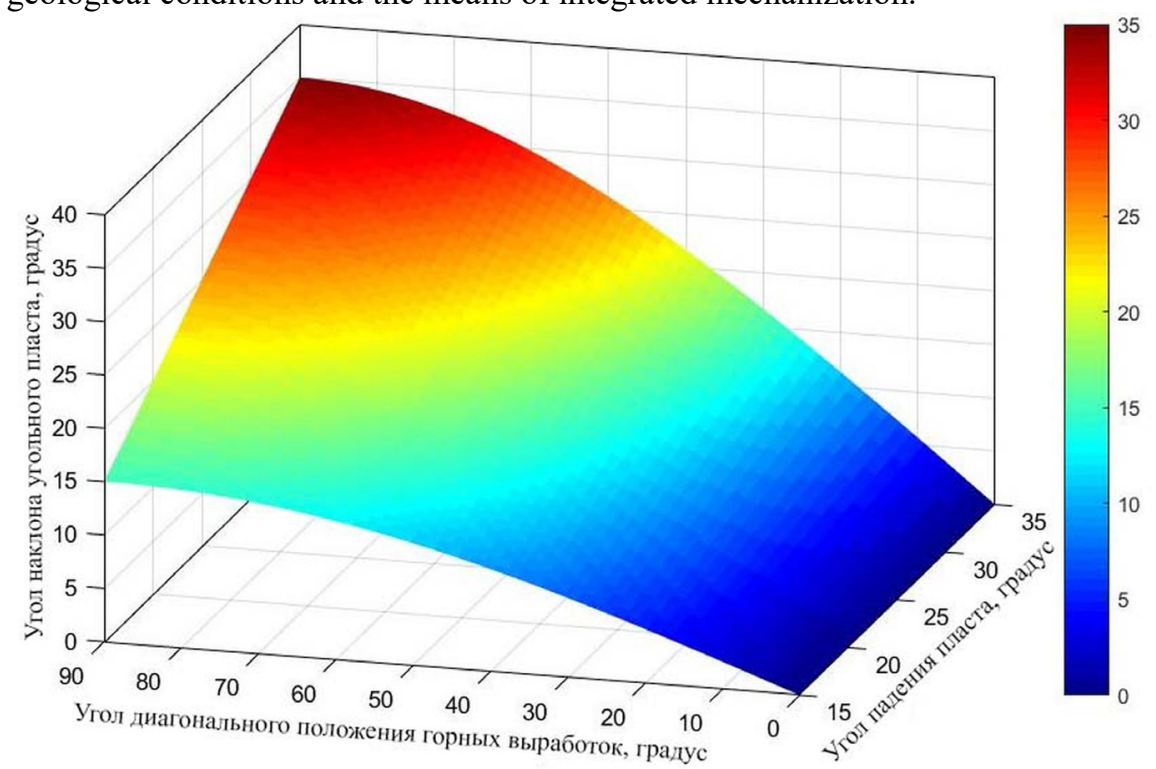

Fig. 3. Scheme for calculating the decrease in the angle of inclination of the mine workings.

\section{Determination of the rational length of the block along the strike}

The rational length along the strike (distance between the chutes along the strike) is determined on the basis of the optimal coordination between the preparatory and working. The time for the development work of a new unit must be linked to the rate of extraction in order to ensure uninterrupted processing of the excavation area, then the duration of mining works is equal to the time of development work. The rational distance between the chutes along the strike is determined and after the conversion, we obtain expressions for determining the distance between the blocks by the following:

$$
L_{c}=\frac{\frac{\gamma \cdot \eta \cdot b \cdot m \cdot a}{Q}+t_{2}-t_{1}}{\frac{\gamma \cdot \eta \cdot b \cdot m \cdot \cos \varphi}{Q}-\frac{\cos \varphi}{v}}
$$

Where: $\gamma$ is density of coal, $\mathrm{t} / \mathrm{m}^{3} ; \eta$ is extraction ratio; $\mathrm{m}$ is thickness of the seam, $\mathrm{m} ; \mathrm{b}$ is the width of the rib pillar, $\mathrm{m} ; \mathrm{L}_{\mathrm{c}}$ is the distance between the blocks along the strike, $\mathrm{m}$; $\mathrm{a}$ is the width of the barrier pillar, $\mathrm{m} ; \varphi$ is the angle between the room axis and the horizontal 
plane, degree; $\mathrm{Q}$ is loading on the working face per day, tons/day; $\mathrm{t}_{1}$ is duration of moving and installing the equipment in a new room, day; $v$ is speed driven of the room, $m / d a y ; t_{2}$ is duration of movement and installation of equipment in a new strip, day.

Justification of the parameters characteristic of the mining and geological conditions of the Uong Bi mine $\left(\gamma=1.6 \mathrm{t} / \mathrm{m}^{3}, \eta=0.8, \mathrm{~m}=3.5 \mathrm{~m}, \mathrm{a}=4 \mathrm{~m}, \mathrm{t}_{1}=3\right.$ days, $\mathrm{t}_{2}=7$ days, $\beta=20$ to $25^{0} ; \mathrm{v}=10 \mathrm{~m} /$ day; ) changes in loading on the face, depending on the width of the rib pillar and the length of the block along the strike, were plotted as shown in 'Figure 4, Figure 5".

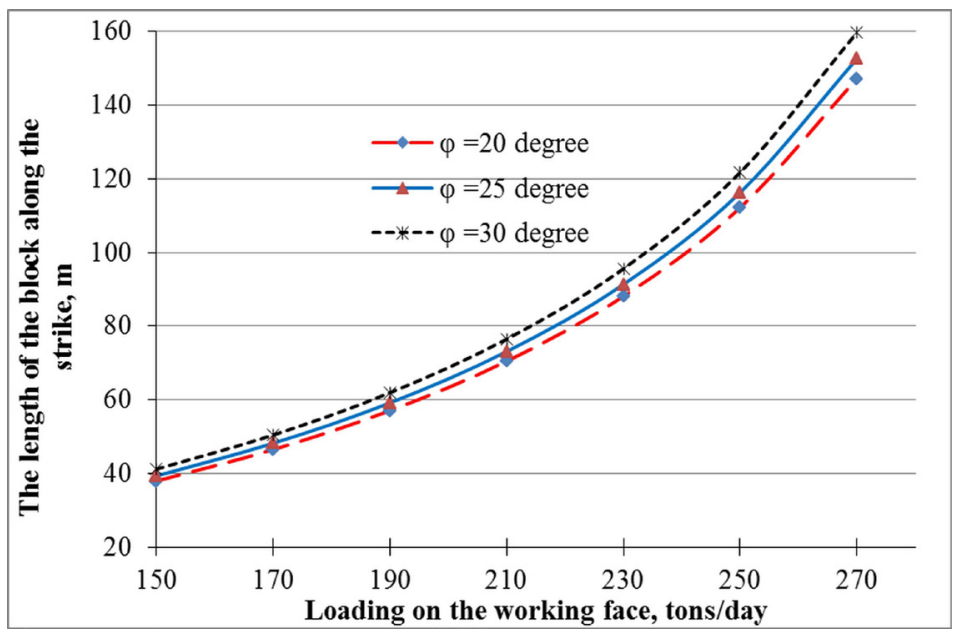

Fig. 4. Dependence of the length of the block along the strike from the load on the working face.

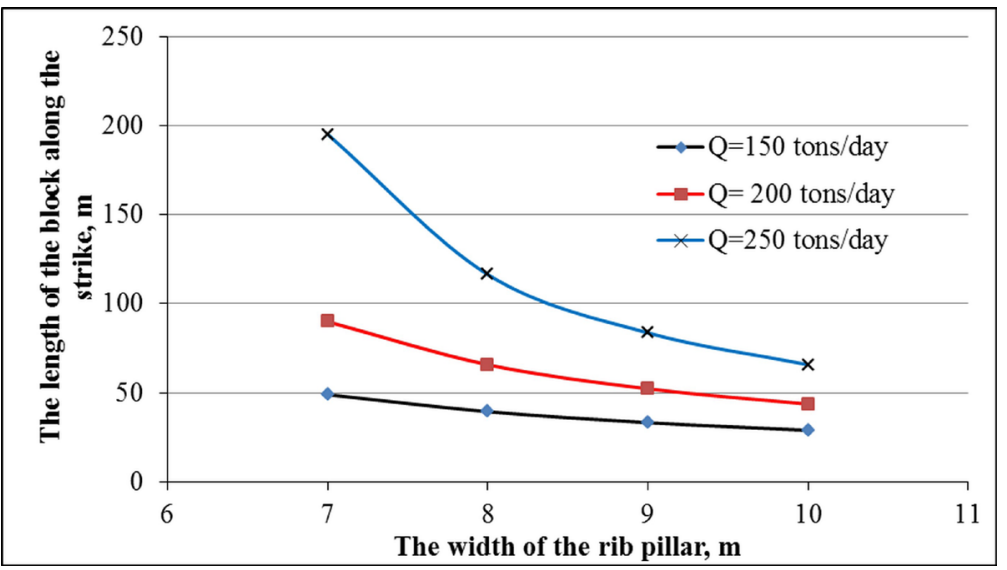

Fig. 5. The nature of the change in the load on the working face as a function of the length of the block along the strike from the width of the rib pillar.

From the analysis of the graphs in "Figure 4 and Figure 5", we can draw the following conclusion that the distance between the chutes along the strike is directly proportional to the load on the face and inversely proportional to the width of the rib pillar. As described above, under conditions of application the room and pillar system at the Uong Bi mine with the length of the block $60 \mathrm{~m}$, it is perfectly consistent with the analysis results in "Figure 4 and Figure 5". In each particular case, the value of the rational length of the column is determined on the basis of the general expression (2). 


\section{Conclusions}

Taking into account the specific mining and geological conditions of the medium thick inclined coal seams, from the experience of using the room and pillar system in the advanced coal-mining countries, we recommend variants of technological schemes for the development of blocks using the room and pillar mining system.

Spatial-planning solutions and parameters of the excavating block, based on intensive working out of the medium thick inclined coal seams with diagonal arrangement of the workings line, are substantiated.

Determination of the rational length of the block along the strike (distance between the chutes along the strike) for strength to provide safety and efficiency in the working of the medium thick inclined coal seams with the room and pillar system in the Quang Ninh coal basin in the dependence of the length of the block (column) on the strike from the load on the working face and the width of the rib pillar.

\section{References}

1. Ordin A.A, and another, Journal of Fundamental and Applied Mining Sciences, Novosibirsk, 1 (2014)

2. L. Van Hau, Journal of Mining Institute, 226, 412 (2017)

3. Remezov A.V and Egorov P.V and Kalinin S.I and Brynko A.F, Kemerovo, 223 (2005)

4. Vitcalov V.G, Pham Duc Thang, Mining informational and analytical bulletin Journal, 10, 113 (2017).

5. Kraskin I.S and A.Vyu Braytsev and SyuVu Shamirov, Coal Journal, 3, 21 (1998)

6. I. B. Tulu, G. S. Esterhuizen, J. A. Sumner, M. A. Sloan, International Journal of Mining Science and Technology, 26, 193 (2016)

7. Nguyen Anh Tuan, Truong Duc Du, Dang Hong Thang, The resulting report of research topics of the Institute of mining science and technology, (Hanoi, 2006)

8. Nguyen Van Vo, Basics of mine design, (Quang Ninh-Viet Nam, 2012).

9. Pham Ngoc Huynh, Nguyen Van Vo, Pham Duc Thang, Opening up and working the deposit by underground mining method, (Quang Ninh-Viet Nam, 2013).

10. Abramkin N.I., Pham Duc Thang, Mining science and technology Journal, 3, 202 (2016)

11. Q. Ninh, The result of work at the Uong bi mine for 2010-2017, (Quang Ninh- Viet Nam, 2017). 